\title{
Reflection of acoustic waves from the boundary or layer of two-phase medium
}

\author{
D.A. Gubaidullin ${ }^{1}$, D.D. Gubaidullina ${ }^{1}$, and Yu.V. Fedorov ${ }^{1, \star}$ \\ ${ }^{1}$ Institute of Mechanics and Engineering KazSC RAS, Department of Continuum Mechanics, 420111, Kazan, Russia
}

\begin{abstract}
The inclined incidence of the acoustic wave on a layer of gas-droplet mixture or bubbly liquid of finite thickness is theoretically investigated. In the case of the incidence of the low-frequency acoustic wave to interface between the pure gas and aerosol or to interface between pure liquid and bubbly liquid the basic laws of reflection and transmission of a wave are established. This circumstance allows us to evaluate the transmission and reflection coefficients, depending on the volume content of inclusions and the angle of incidence of the acoustic wave. In particular, for the interface between pure gas and aerosol analytical expressions of the critical angle of wave incidence at which reflection coefficient becomes zero are obtained, i.e. thus there is a complete passage of the acoustic wave through the interface. It is established that the increase of the angle of incidence of the acoustic wave on the boundary or layer of aerosol causes: first, either to increase or to decrease of the reflection coefficient at low frequencies, and second, to appearance of additional minima depending on the reflection coefficient from frequency of disturbances related to the difference of speed of sound and density of the medium.
\end{abstract}

\section{Introduction}

The problem of wave propagation in complex layered media containing layers of bubbly liquid or suspension of gas with particles is topical. This is due to both natural phenomena, such as, for example, near-bottom or subsurface layers containing gas bubbles in seas and lakes [1-5], layers of fog, dusty gas, containing in atmosphere [6-8], and engineering processes $[9,10]$. The design and parameters of a combined panel consisting of two glasses or polycarbonates with a gap between them filled with a bubbly fluid is described in [10]; this panel is used for the simultaneous information protection in the acoustic, optical, and electromagnetic technical channels.

Among experimental studies we notice [11], where the two-layer water - water with air bubbles - air medium was considered and the reflection coefficient was determined in the case of the normal acoustic-wave incidence from the direction of the water. In [12] the two-layer water water with air bubbles - water medium was considered and the coefficient of transmission through a bubble screen was experimentally estimated. In [13] the propagation of a pulsed disturbance through a three-layer specimen placed in a fluid and consisting of a bubbly gel layer bounded by polycarbonate layers was experimentally investigated. The comparison of the theory with the experimental data obtained in [13] was made in [14] and good agreement was found to exist.

The main approaches to an investigation of the acoustics of bubbly fluids and gas-particle suspension can be found in the well known books [15-18], while paper [19] gives an overview of certain publications.

The problem of small disturbance propagation in a liquid with gas-vapor bubbles in some or another formulation was studied in [20-33].

In this study reflection of acoustic waves from the boundary or layer of two-phase medium is investigated.

\subsection{Reflection and transmission coefficients}

To determine the transmission and reflection from twolayer medium coefficients, a diagram of which is shown in fig. 1, the following method of calculations is used. According to [34], the result of the reflection and transmission of a plane monochromatic wave exp $i(\omega t-K z)$ from multi-layer media will be plane waves $R \exp i(\omega t+K z)$ and $T \exp i(\omega t-K z)$, where $R$ and $T-$ reflection and transmission of the waves coefficients, which are defined through layers impedances $Z_{j}$ and input impedances of layers borders $Z_{j}^{\text {in }}$. For a two-layer medium reflection and transmission coefficients are of the form

$$
R=\frac{Z_{2}^{i n}-Z_{1}}{Z_{2}^{i n}+Z_{1}}, T=\prod_{j=1}^{2}\left(\frac{Z_{j}^{i n}+Z_{j}}{Z_{j}^{i n}+Z_{j+1}} \exp i K_{j} s_{j}\right)
$$

where

$$
\begin{gathered}
Z_{j}=\rho_{j} \frac{\omega}{K_{j}}, Z_{2}^{i n}=\frac{Z_{3}-i Z_{2} \tan \left(K_{2} s\right)}{Z_{2}-i Z_{3} \tan \left(K_{2} s\right)} Z_{2} \\
i^{2}=-1, j=1,2,3
\end{gathered}
$$




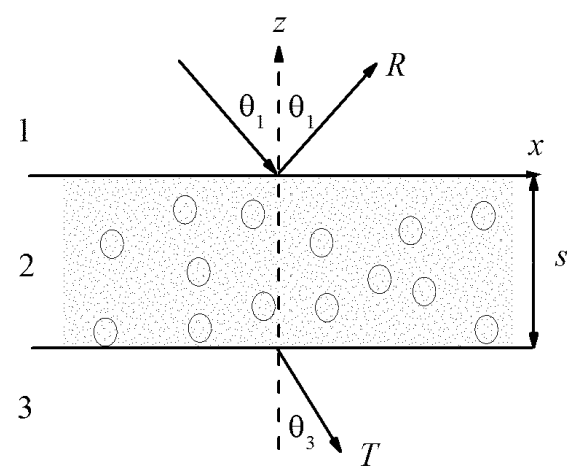

Figure 1. Scheme of the media

Here $s$ - the thickness of the bubble layer, $K$ - wave number, $\omega$ - frequency of disturbances, $\rho$ - density of the layer. For a homogeneous layer the wave number is defined as $K_{j}=\omega / C_{j}$, where $C_{j}$ - the speed of sound in the $j$-th layer.

\subsection{Complex wave number for bubbly liquid}

Since in the second layer bubble liquid will be considered, i.e., non- homogeneous and absorbing medium, to determine, in general, a complex wave number $K_{2}=K_{b l}$ the relation obtained in [21] will be used. This dependence of a complex wave number on the disturbances frequency is given by

$$
\left(\frac{K_{b l}}{\omega}\right)^{2}=\frac{1}{C_{f}^{2}}+\frac{3 \alpha_{2} \alpha_{1} \rho_{1} Q}{3 \gamma_{2} p_{1}-Q S}
$$

where

$$
\begin{gathered}
Q=1+\phi, \phi=3\left(\gamma_{2}-1\right) \frac{y \operatorname{coth} y-1}{y^{2}}, y=\left(-\frac{i \omega a^{2}}{\kappa_{2}}\right)^{1 / 2} \\
S=\frac{i \omega a^{2} h \rho_{1}}{1+h t}, h=\frac{4 v_{1}}{a^{2}}-i \omega, t=\frac{a}{C_{1} \alpha_{2}^{1 / 6}}
\end{gathered}
$$

Here, the subscript 1 refers to the parameters of the liquid phase, index 2 - to the parameters of the bubbles. The parameters $\alpha$ determine the volume content, $\rho_{1}$ - liquid density, $\rho_{2}-$ density of gas in bubbles, $p_{1}$ - pressure, $C_{f}$ - frozen velocity, is almost equal to the speed of sound $C_{1}$ in pure liquid, $\gamma$ - the adiabatic index, $\kappa$ - coefficient of the gas thermal conductivity, $v_{1}-$ the kinematic viscosity of the fluid, $a$-radius of bubbles.

Let us briefly explain the derivation of equation (2). A system of differential equations that determines the propagation of an acoustic wave in the medium under consideration is recorded. Then the system of equations is linearized. The solution of the obtained linear system of equations in the form of progressive waves is considered: $A_{\phi} \exp (i(K x-\omega t))$, where $x$ - coordinate, $A_{\phi}$-amplitude, $K$ - wave number, $t$-time. The dispersion equation (2) is obtained from the condition of the existence of uniqueness of a non-trivial solution for a linear system of equations. A detailed derivation of this relation is presented in [21].

\subsection{Complex wave number for gas-drops suspension}

Similarly an expression of the wave number for a gasdrops suspension, which has a view [18] is derived:

$$
\left(\frac{C_{1} K_{g l}}{\omega}\right)^{2}=V(\omega) D(\omega), V(\omega)=1+\frac{m}{1-i \omega \tau_{v}^{*}}
$$

where

$$
D(\omega)=1+\left(\gamma_{1}-1\right) \frac{t_{d}}{1+t_{d}}, t_{d}=\frac{m c_{p 2} / c_{p 1}}{1-i \omega \tau_{T}^{*}}
$$

$$
\begin{gathered}
\tau_{v}^{*}=\tau_{v}\left(1+\frac{1-i}{\sqrt{2}}\left(\omega \tau_{\mu_{1}}\right)^{\frac{1}{2}}\right)^{-1}, \tau_{v}=\frac{2 \rho_{2} a^{2}}{9 \mu_{1}}, \tau_{\mu_{1}}=\frac{\rho_{1} a^{2}}{\mu_{1}} \\
m=\frac{\alpha_{2} \rho_{2}}{\alpha_{1} \rho_{1}}, \tau_{T}^{*}=\tau_{T 2}^{*}+\frac{m c_{p 2}}{c_{p 1}} \tau_{T 1}^{*}, \tau_{T 1}^{*}=\frac{\alpha_{1} \tau_{\lambda_{1}}}{3 \alpha_{2}\left(1+z_{1}\right)} \\
z_{1}=\frac{1-i}{\sqrt{2}}\left(\omega \tau_{\lambda_{1}}\right)^{\frac{1}{2}}, \tau_{T 2}^{*}=\frac{\tau_{\lambda_{2}}}{3} \frac{3 z_{2}-\left(3+z_{2}^{2}\right) \tanh z_{2}}{z_{2}^{2}\left(\tanh z_{2}-z_{2}\right)} \\
z_{2}=\frac{1-i}{\sqrt{2}}\left(\omega \tau_{\lambda_{2}}\right)^{\frac{1}{2}}, \tau_{\lambda_{j}}=\frac{a^{2}}{\kappa_{j}}, \kappa_{j}=\frac{\lambda_{j}}{\rho_{j} c_{p j}}, j=1,2
\end{gathered}
$$

The subscript 1 refers to the parameters of the carrier phase, index $2-$ to the parameters of the droplets. The parameters $\alpha$ determine the volume content, $\rho$-density, $C_{1}-$ sound velocity, $v$ - the kinematic viscosity, $\lambda$ - coefficient of thermal conductivity, $a$-radius of drops.

\section{Results of the calculations}

Let's consider the air mixtures with drops of water and water with air bubbles and calculate the phase velocity in these mixtures. The figures $2 \mathrm{a}, \mathrm{b}$ show the results of comparing of dependencies of the phase velocity on the disturbance frequency $f=\omega / 2 \pi$ for different values of the volume content of droplets (a) and bubbles (b). The calculations are carried by using formulas (2), (3). As for a gas suspension and for a bubble liquid in a large range of low frequencies, the phase velocity is not changed and possesses a certain equilibrium value, which depends on the volume content of the inclusions. With this increase in the volume content of inclusions, this equilibrium value decreases. At high frequencies, the phase velocity for these two media tends to the speed of sound in the carrier phase.

Analytic expressions of the equilibrium velocity for a gas suspension and a bubble liquid are obtained by passing to the limit $\omega \rightarrow 0$ in formulas (2),(3) and are written accordingly

$$
\begin{gathered}
C_{b l}^{e}=\left(\frac{1}{C_{1}^{2}}+\frac{\rho_{1} \alpha_{1} \alpha_{2}}{p_{1}}\right)^{-\frac{1}{2}} \\
C_{g l}^{e}=C_{1}\left(\frac{\gamma_{e}}{\alpha_{1}(1+m) \gamma_{1}}\right)^{\frac{1}{2}}, \gamma_{e}=\frac{c_{p 1}+m c_{p 2}}{c_{p 1} / \gamma_{1}+m c_{p 2}}
\end{gathered}
$$

Let us consider the fall of a low-frequency acoustic wave at different angles to the interface between pure air and a mixture of air with water droplets on the air side when the speed of sound in the gas-droplet medium takes 

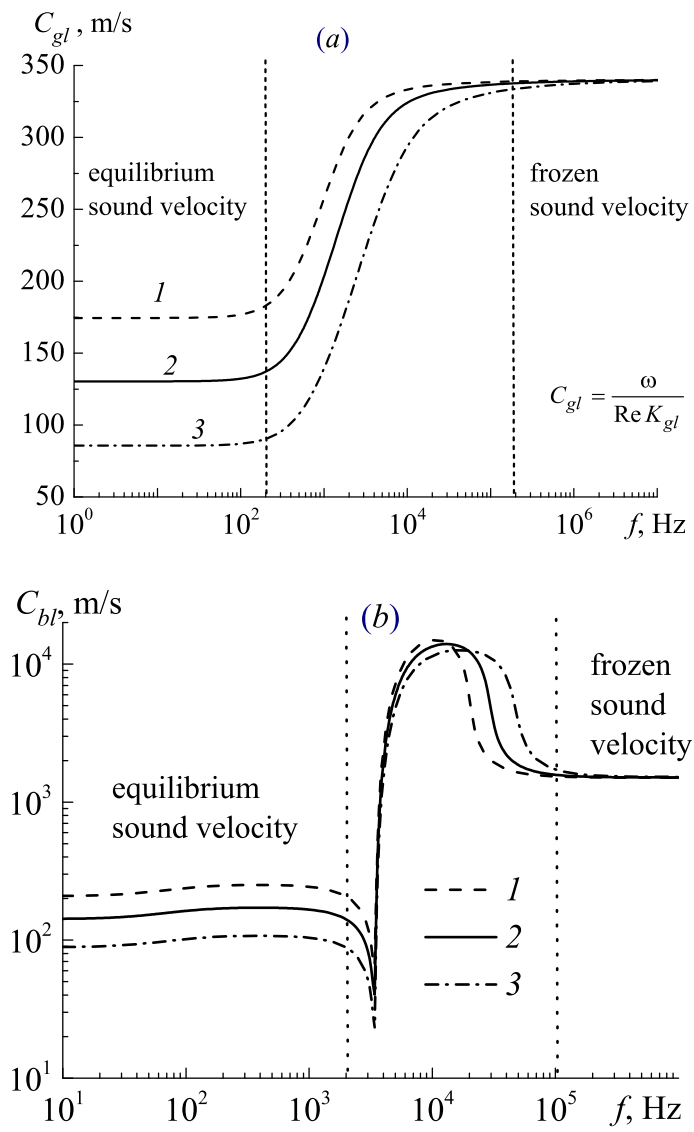

Figure 2. Dependences of phase velocity on the frequency of perturbation for mixture of air with water drops (a) and water with air bubbles (b) at various volume contents of inclusions: 1 $0.0023,2-0.005,3-0.013$; radius of drops $-5 \mathrm{mkm}$, radius of bubbles $-1 \mathrm{~mm}$

on an equilibrium value $C_{g l}^{e}(5)$. The reflection and transmission coefficients are written as the following formulas.

$$
\begin{gathered}
R=\frac{Z_{g l}-Z_{1}}{Z_{g l}+Z_{1}}, Z_{g l}=\frac{\rho_{g l} C_{g l}^{e}}{\cos \theta_{g l}}, Z_{1}=\frac{\rho_{1} C_{1}}{\cos \theta_{1}} \\
\theta_{g l}=\arcsin \left(\frac{C_{g l}^{e}}{C_{1}} \sin \theta_{1}\right), \rho_{g l}=\alpha_{1} \rho_{1}+\alpha_{2} \rho_{2} \\
T-R=1
\end{gathered}
$$

The figures $3 \mathrm{a}$ and $\mathrm{b}$ show the dependencies of the reflection coefficient on the volume content of water droplets (a) and the angle of incidence of the acoustic wave (b). Lets pay attention to the fact that for a value of the angle $\theta_{1}=$ 0 , the dependence of the reflection coefficient on the volume content of droplets (Figure $3 \mathrm{a}$ ) is monotonic, and the acoustic wave begins to be reflected only at the values of the volume content of the droplets $\alpha_{2}>10^{-3}$. However, if we increase the angle of incidence, then the dependence $\left|R\left(\alpha_{2}\right)\right|$ becomes nonmonotonic, and the wave begins to be reflected from the boundary already when $\alpha_{2}<0.001$. Another important conclusion is that for certain values of the angle of incidence and the volume content of drops, the reflection coefficient has zero values (Figs. $3 \mathrm{a}$ and b). In this case, the wave passes completely (without reflection) through the interface. Below is the equation for the critical angle at which this situation is realized.

$$
\sin \theta_{1}^{*}=\sqrt{\frac{\left(\rho_{g l} C_{g l}^{e}\right)^{2}-\rho_{1}^{2} C_{1}^{2}}{\left(C_{g l}^{e}\right)^{2}\left(\rho_{g l}^{2}-\rho_{1}^{2}\right)}}
$$

It should be noted that the extremum of the reflection coefficient is possible only if the density of the gas droplet medium differs from the density of pure gas $\rho_{g l} \neq \rho_{1}$.
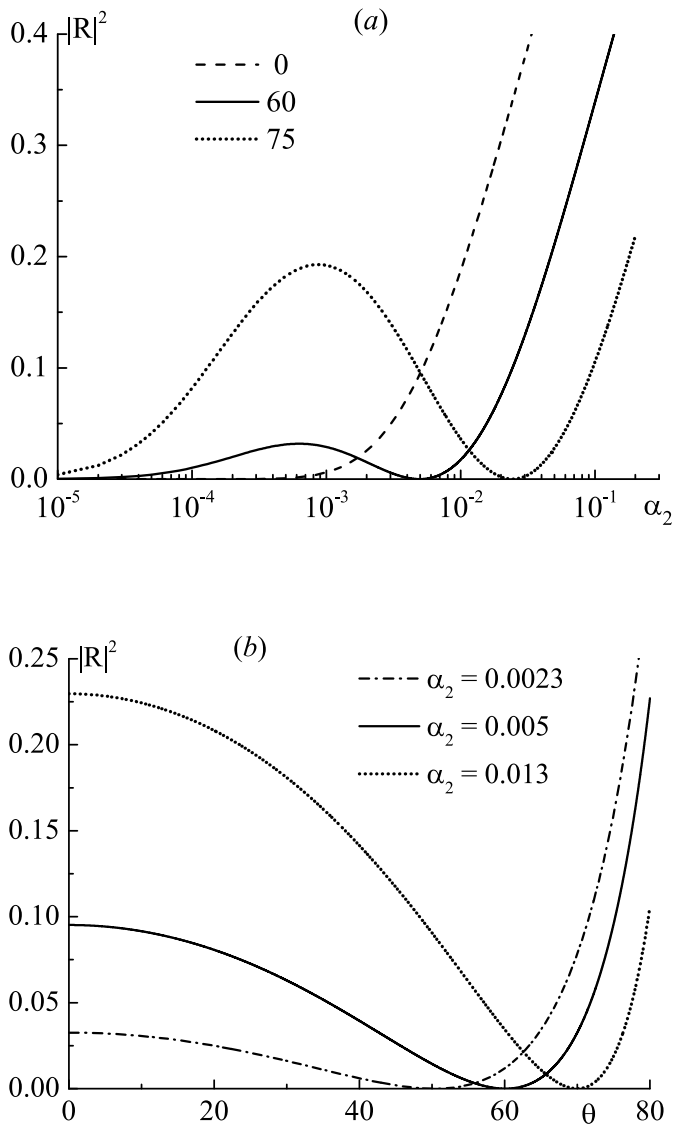

Figure 3. Dependences of reflection coefficient on volume content of drops at various angle of incidence (a) and on angle of incidence at various volume content of drops (b)

The figures 4 and 5 show the results of comparing of the dependencies of the reflection coefficient on the disturbance frequency $f=\omega / 2 \pi$. Curve 1 is constructed for the medium air / gas-droplet mixture. Comparison of this curve 1 with theoretical data 4 is shown. The curves 2, 3 correspond to the media air / gas-drop mixture / air and air / gas-droplet / water mixtures, respectively. The radius $a$ of the water droplets was $5 \mu \mathrm{m}$, the volume content $\alpha_{2}=0.005$, the acoustic wave fell from the side of air at an angle $\theta_{1}=0$, the thickness of the gas-droplet mixture layer $s=0.3 \mathrm{~m}$. The nature of the curve 1 of the reflection coefficient corresponds to the character of the curve of sound speed in the gas droplet mixture. At frequencies up to $200 \mathrm{~Hz}$ and above $20000 \mathrm{~Hz}$, the reflection coefficient, like the speed of sound, does not change its equilibrium value and is approximately 0.1 and 0.44 , respec- 


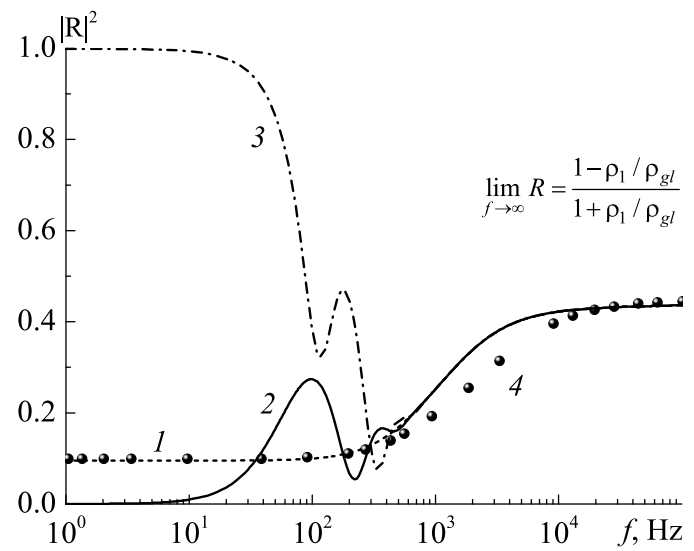

Figure 4. Results of calculations of the reflection coefficient for media: 1 - air / air with water drops, 2 - air / air with water drops / air, 3 - air / air with water drops / water, 4 - theoretical data [16]; the thickness of the layer $s$ is $0.3 \mathrm{~m}, a=5 \mu \mathrm{m}, \alpha_{2}=$ 0.005

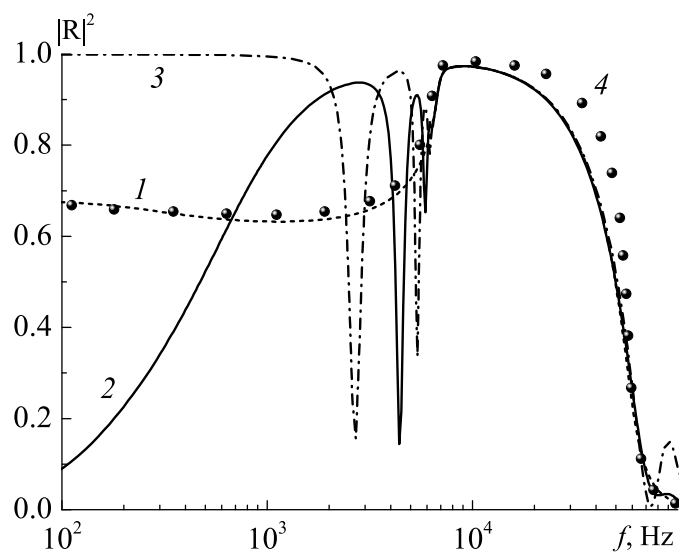

Figure 5. Results of calculations of the reflection coefficient for media: 1 - water / bubbly liquid, 2 - water / bubbly liquid / water, 3 - water / bubbly liquid / air, 4 - theoretical data [16]; the thickness of the layer $s$ is $0.015 \mathrm{~m}, a=0.5 \mathrm{~mm}, \alpha_{2}=0.005$

tively. At frequencies $f>600 \mathrm{~Hz}$, the curves 2,3 go to curve 1 . This means that the influence of the third medium decreases and the reflection coefficient is of the same nature as if the wave fell on the interface between the air and the gas-droplet mixture. An analogous character of the reflection coefficient is also observed for the water/bubble liquid boundary.

In the example of interface of the medium air-gas suspension, the effect of the angle of incidence of the wave on the dependencies of the reflection coefficient on the disturbance frequency is shown in the figure 6 . At frequencies up to $200 \mathrm{~Hz}$, where the sound speed $C_{g l}$ assumes an equilibrium value $C_{g l}^{e}$, the nature of the reflection coefficient corresponds to the nature of the reflection coefficient in the low-frequency region calculated by us earlier. It is seen that for certain angles of incidence of the wave, a nonmonotonic dependence of the reflection coefficient on the frequency of the disturbances is observed. You can evalu- ate the position of extremums using the following formula.

$$
\begin{gathered}
\min |R(\omega)| \Rightarrow \sin ^{2} \theta_{1}=\frac{\left(\sigma^{2}-1\right) n^{2}+m^{4}}{m^{4}-1}- \\
-\frac{\sqrt{\left(m^{4}\left(1+\sigma^{2}\right)^{2}-4 \sigma^{2}\right) n^{4}+2 m^{4}\left(\sigma^{2}-1\right) n^{2}+m^{4}}}{m^{4}-1} \\
\sigma=\frac{\mathfrak{J} K_{g l}}{\mathfrak{R} K_{g l}}, n=\frac{C_{1}}{C_{g l}}, m=\frac{\rho_{g l}}{\rho_{1}}
\end{gathered}
$$

If $\omega \rightarrow 0$ then $\sigma \rightarrow 0, n \rightarrow C_{1} / C_{g l}^{e}$ and formula (10) $\rightarrow$ (9). If $\omega \rightarrow \infty$ then $\sigma \rightarrow 0, n \rightarrow 1$ and $\theta_{1} \rightarrow \pi / 2$. From the estimates, we come to the conclusion that the nonmonotonicity of the reflection coefficient from the disturbance frequency appear when the angle of incidence of the wave is in the interval $\left(\theta_{1}^{*}, \pi / 2\right)$ and the density of the gas droplet medium $\rho_{g l}$ is bigger than the density of the pure gas $\rho_{1}$.

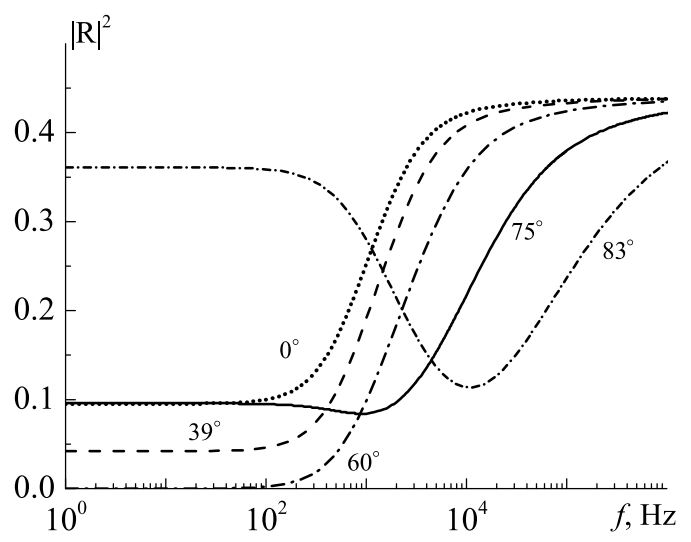

Figure 6. Dependencies of reflection coefficient on the frequency at various angle of incidence of wave for interface "air air with water drops"

\section{Conclusion}

1. At normal incidence of the acoustic wave, the nature of the reflection coefficient at low frequencies for the layers containing the gas-droplet mixture or the bubble liquid does not qualitatively differ, but at high frequencies the reflection coefficient for the bubble liquid layer tends to zero, and for the gas-suspension layer with volume contents of inclusions $\alpha_{2}>0.2 \times 10^{-3}$ has a finite nonzero value.

2. With an inclined incidence of the acoustic wave, the difference is at low frequencies, namely, the reflection coefficient for the boundary or the layer of the gas-droplet mixture can both increase and decrease, while for the bubble liquid the reflection coefficient only increases with increasing angle of incidence of the wave.

3 . For the boundary or the layer of the gas-droplet mixture, a minimum of the reflection coefficient may appear, depending on the frequency of the disturbances in a certain range of the angle of incidence of the wave and at certain values of the volume content of droplets, which is associated mainly with the difference in the densities of the gas-droplet mixture and pure gas. 


\section{Acknowledgment}

This work was supported by the Russian Science Foundation (grant 15-11-20022).

\section{References}

[1] V.Sh. Shagapov, V.V. Sarapulova, Acoustical Physics 61, 37 (2015)

[2] D.A. Gubaidullin, Yu.V. Fedorov, Fluid Dynamics 52, 107 (2017)

[3] V.A. Gusev, O.V. Rudenko, Acoustical Physics 61, $152(2015)$

[4] D.A. Gubaidullin, Yu.V. Fedorov, Doklady Physics 61, 499 (2016)

[5] D.A. Gubaidullin, D.D. Gubaidullina, Yu.V. Fedorov, J. Phys.: Conf. Ser. 789, 012016 (2017)

[6] J.E. Cole, R.A. Dobbins, J. Atmos. Sci. 28, 202 (1971)

[7] G.A. Davidson, J. Atmos. Sci. 32, 2201 (1975)

[8] R. Ishii, H. Matsuhisa, J. Fluid Mech. 130, 259 (1983)

[9] V. Leroy, A. Strybulevich, M. Lanoy, F. Lemoult, A. Tourin, J.H. Page, Phys. Rev. B. 91, 020301 (2015)

[10] O.B. Zelmanskiy, S.N. Petrov, A.A. Kazeka, Doklady BGUIR 78, 30 (2013)

[11] K. Lee, B.K. Choi and S.W. Yoon, Journal of the Korean Physical Society 40, 256 (2002)

[12] T.M. Tien, Sound propagation through a bubble screen at finite gas-volume fraction (Taiwan: National Cheng Kung University, 2001)

[13] V. Leroy, A. Strybulevych, J.H. Page, M.G. Scanlon, J. Acoust. Soc. Amer. 123, 1931 (2008)

[14] R.I. Nigmatulin, D.A. Gubaidullin, A.A. Nikiforov, Doklady Physics 59, 286 (2014)

[15] R.I. Nigmatulin, Dynamics of multiphase media (Moscow: Nauka, 1987)

[16] S. Temkin, Suspension acoustics: an introduction to the physics of suspensions (Cambridge: Cambridge University Press, 2005)
[17] V.E. Nakoryakov, B.G. Pokusaev, I.R. Shreiber, Wave Dynamics of Gas- and Vapor - Liquid Media (Moscow: Energoatomizdat, 1990)

[18] D.A. Gubaidullin, Dynamics of two-phase gasvapor-droplet media (Kazan: Kazan mathematical society, 1998)

[19] A.Yu. Varaksin, High Temperature 51, 377 (2013)

[20] R.N. Gafiyatov, D.A. Gubaidullin, A.A. Nikiforov, Fluid Dynamics 48, 366 (2013)

[21] D.A. Gubaidullin, Yu.V. Fedorov, Journal of Applied Mathematics and Mechanics 77, 532 (2013)

[22] D.A. Gubaidullin, R.N. Gafiyatov, J. Phys.: Conf. Ser. 567, 012020 (2014)

[23] D.A. Gubaidullin, Yu.V. Fedorov, Acoustical Physics 62, 179 (2016)

[24] T.G. Leighton, J. Jiang, K. Baik, J. Acoust. Soc. Amer. 131, 2413 (2012)

[25] D.A. Gubaidullin, Yu.V. Fedorov, Fluid Dynamics 50, 61 (2015)

[26] A. Prosperetti, Annu. Rev. Fluid Mech. 49, 221 (2017)

[27] A. Prosperetti, Interface Focus. 20140024 (2015) doi:10.1098/rsfs.2015.0024

[28] D. Fuster, F. Montel, J. Fluid Mech. 779, 598 (2015)

[29] D.A. Gubaidullin, D.D. Gubaidullina, Yu.V. Fedorov, J. Phys.: Conf. Ser. 567, 012011 (2014)

[30] D.A. Gubaidullin, A.A. Nikiforov, R.N. Gafiyatov, High Temperature 53, 240 (2015)

[31] D.A. Gubaidullin, A.A. Nikiforov, R.N. Gafiyatov, J. Phys.: Conf. Ser. 669, 012019 (2016)

[32] D.A. Gubaidullin, R.N. Gafiyatov, J. Phys.: Conf. Ser. 789, 012019 (2017)

[33] D.A. Gubaidullin, D.D. Gubaidullina, Yu.V. Fedorov, J. Phys.: Conf. Ser. 669, 012011 (2016)

[34] L.M. Brekhovskikh, O.A. Godin, Acoustics of Layered Media (Moscow: Nauka, 1989) 